\title{
Vorticity of viscous electronic flow in graphene
}

\author{
Sven Danz ${ }^{1}$ and Boris N. Narozhny ${ }^{1,2}$ \\ ${ }^{1}$ Institut für Theorie der kondensierten Materie, Karlsruhe Institute of Technology, 76128 Karlsruhe, Germany \\ ${ }^{2}$ National Research Nuclear University MEPhI (Moscow Engineering Physics Institute), 115409 Moscow, Russia
}

(Dated: June 1, 2020)

\begin{abstract}
In ultra-pure materials electrons may exhibit a collective motion similar to the hydrodynamic flow of a viscous fluid, the phenomenon with far reaching consequences in a wide range of many body systems from black holes to high-temperature superconductivity. Yet the definitive detection of this intriguing behavior remains elusive. Until recently, experimental techniques for observing hydrodynamic behavior in solids were based on measuring macroscopic transport properties, such as the "nonlocal" (or "vicinity") resistance, which may allow alternative interpretation. Earlier this year two breakthrough experiments demonstrated two distinct imaging techniques making it possible to "observe" the electronic flow directly. We demonstrate that a hydrodynamic flow in a long Hall bar (in the absence of magnetic field) exhibits a nontrivial vortex structure accompanied by a sign-alternating nonlocal resistance. An experimental observation of such unique flow pattern could serve a definitive proof of electronic hydrodynamics.
\end{abstract}

Traditional fluid mechanics ${ }^{1}$ describes long-distance properties of conventional (e.g., water, oil, etc.) and quantum (e.g., ${ }^{3} \mathrm{He}$ ) fluids equally well $\frac{213}{3}$. The key feature uniting these diverse many body systems is the short-ranged or collision-like nature of interactions between the constituent particles that conserve momentum. In the simplest case of a dilute gas (e.g., air) the hydrodynamic equations can be derived from the kinetic theory ${ }^{4}$. The resulting theory is however more general than the derivation: the hydrodynamic equations provide a universal description applicable to any system within the same symmetry class.

The usual theory of the electron transport in solids also relies on the kinetic theory ${ }^{\sqrt[5]{5}}$, but with momentumrelaxing scattering processes typically dominating over the momentum-conserving electron-electron interaction. Unlike the conventional fluids, electrons in solids exist in the environment created by a crystal lattice where scattering off either lattice imperfections (or "disorder") or lattice vibrations ("phonons") does not conserve momentum. At length scales exceeding the mean free path $\ell_{\text {dis }}$ (or $\ell_{\mathrm{e}-\mathrm{ph}}$ ) the electrons exhibit diffusive motion ${ }^{215}$. In relatively small, mesoscopic samples of the size smaller than (or comparable to) the mean free path, $L \lesssim \ell_{\mathrm{dis}}, \ell_{\mathrm{e}-\mathrm{ph}}$, the electrons can cross the sample ballistically $\sqrt{617}$.

In contrast, should one manage to fabricate a sample, where the momentum-conserving electron-electron interaction were the dominant scattering process, the macroscopic flow of electrons would be hydrodynamic ${ }^{810}$. Envisioned by Gurzhi10 long time ago, this idea got traction only with the emergence of ultra-pure materials 1120 . Yet, while it has been established that transport properties of such systems deviate significantly from the traditional expectation ${ }^{5}$, the "hydrodynamic" interpretation of the observed results may still be considered as controversial. In particular, the nonlocal resistance (a tool often used to uncover hydrodynamic transport $\frac{14115(20)}{4}$ has been extensively studied in the context of ballistic transport in multiterminal measurements in the presence $e^{21} \sqrt{23}$ or absence $e^{24} \sqrt{27}$ of an external magnetic field. Thus the hydrodynamic flow can be distinguished from a ballistic one ${ }^{28}$ by employing additional measurements: the nonlocal resistance should be supplemented by measurements of its temperature dependence $e^{15}$ and even imaging of the Poiseuille velocity profile has to be supplemented by measurements of additional quantities $\$ 11$.

Recently two breakthrough experiments demonstrated two distinct imaging techniques allowing to "observe" the electronic flow directly 7/11/12. This makes it possible to study a distinct feature of the hydrodynamic flow - vorticity - in laboratory experiments. The hydrodynamic vortices (otherwise known as eddies or whirlpools) were previously invoked to explain the observed negative nonlocal resistance in graphene 20129130, but so far have not been directly imaged. In this paper, we solve the unconventional hydrodynamic equations in graphene $e^{31 / 32}$ in a realistic Hall bar geometry (away from neutrality) and demonstrate that the electronic flow forms a unique pattern exhibiting multiple vortices. Alternation in spinning direction of these vortices results in a sign-alternating nonlocal resistance ${ }^{33}$. These features could be used to distinguish the hydrodynamic flow (to which they are unique) from ballistic propagation.

Within linear response and in the case of stationary flow, the Navier-Stokes equation (A1) in graphene away from charge neutrality (i.e., the system considered in the imaging experiments of Refs. 7/11) takes the form9129132

$$
v_{g}^{2} \boldsymbol{\nabla} P=v_{g}^{2}[\eta \Delta \boldsymbol{u}+e n \boldsymbol{E}]-\frac{\mu n \boldsymbol{u}}{\tau_{\mathrm{dis}}},
$$

where $\eta$ is the shear viscosity $1420 \mid 34, n$ is the carrier density, $\boldsymbol{E}$ is the electric field, $v_{g}$ is the Fermi velocity, $P$ is the thermodynamic pressure, $\mu$ is the chemical potential, and $\tau_{\text {dis }}$ is the disorder mean free time, see Appendix $\mathrm{A}$ for details. At high enough densities, the electric current is proportional to the hydrodynamic velocity, $\boldsymbol{J}=e n \boldsymbol{u}$. In the rest of the paper we analyze this equation numerically and establish the local flow patterns in experimentally relevant geometries.

To verify our numerical methods (see Appendix B), we 


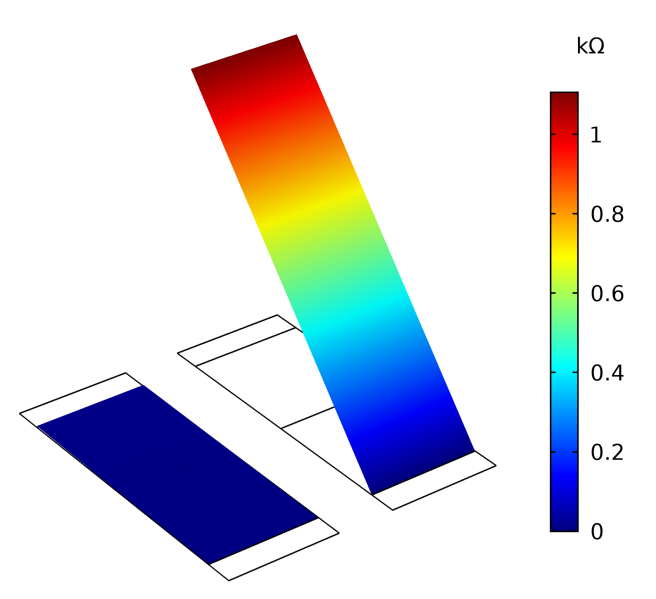

FIG. 1: Ballistic-to-diffusive crossover in inviscid electronic flow in doped graphene (cf. Fig. 2 of Ref. 17). The two plots show the electrochemical potential $\Phi$ normalized by the driving current $I$ for $\ell_{\text {dis }}=26 \mu \mathrm{m}$ (ballistic propagation; left) and $\ell_{\mathrm{dis}}=0.15 \mu \mathrm{m}$ (diffusive flow; right). The calculations were performed at $T=4 \mathrm{~K}, n=10^{12} \mathrm{~cm}^{-2}$, and $L=25 \mu \mathrm{m}$. The left plot does not show the step-like contact voltage drops ${ }^{7 / 35}$ since the contact resistance ${ }^{36}$ was not included in the calculation.

first consider the hydrodynamic equation (1) in the absence of viscosity, $\eta=0$. In this case, Eq. (1) describes the usual Ohmic flow of electrons. By varying the ratio of the mean free path, $\ell_{\text {dis }}=v_{g} \tau_{\text {dis }}$, to the sample length $L$ within the two-terminal measurement scheme we observe the ballistic-to-diffusive crossover ${ }^{7}$, see Fig. 1. Combining the pressure gradient and the electric field into the gradient of the electrochemical potentia 2937 , we follow the change of the latter along the rectangular sample placed between the two contacts. Choosing the mean free path to exceed the sample length $\left(\ell_{\mathrm{dis}}=26 \mu \mathrm{m}\right.$, see Ref. 7) we observe the almost flat profile of the electrochemical potential across the sample indicative of ballistic propagation (cf. the effect of electric field expulsion $\left.{ }^{35}\right)$, see the left panel in Fig. 1. In contrast, a system with a smaller mean free path $\left(\ell_{\text {dis }}=0.15 \mu \mathrm{m}\right)$ exhibits a linear variation of the electrochemical potential typical of the diffusive transport, see the left panel in Fig. 1. Not surprisingly, this behavior fully agrees with experimental observations (see Fig. 2 of Ref. 7).

Viscous flow in the same two-terminal geometry is expected to exhibit a Poiseuille-like velocity profile ${ }^{9|11 / 38| 39}$. The precise form of the profile is determined by the interplay of the viscosity, disorder mean free time, and boundary conditions. The parabolic profile ${ }^{1}$ corresponds to a pure liquid $\left(\tau_{\text {dis }} \rightarrow \infty\right)$ with the "no-slip" boundary conditions ( $\boldsymbol{u}=0$ at the sample edges). The latter were recently shown to be inapplicable in graphene 40 ; instead, one should apply a more general boundary condition, Eq. (B1), characterized by the temperature-dependent "slip length". This quantity was recently measured in

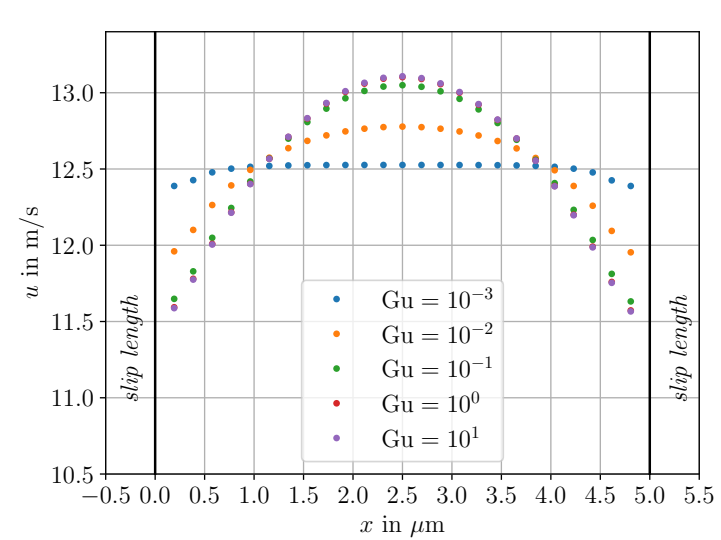

FIG. 2: Flow velocity profile in a viscous electronic flow in a strip geometry $(W=5 \mu \mathrm{m}, L=10 \mu \mathrm{m})$. The profiles were calculated with the "slip boundary condition" with the slip length ${ }^{11]} \zeta=0.5 \mu \mathrm{m}$. The color-coded datasets correspond to different values of the Gurzhi number (2).

graphene ${ }^{11}$ to be $\zeta \approx 500 \mathrm{~nm}$ at $T=75 \mathrm{~K}$.

To describe the mutual effect of disorder and viscosity on the electronic flow we introduce a "Gurzhi number"

$$
\mathrm{Gu}=\frac{\nu \tau_{\text {dis }}}{l^{2}}, \quad \nu=\frac{v_{g}^{2} \eta}{\mathcal{W}}
$$

where $\nu$ is the kinematic viscosity $114420134, \mathcal{W}$ is the enthalpy [see Eq. [A2)] and $l$ is a typical length scale in the problem (e.g., the width of the graphene strip) in analogy to the standard composition of the Reynolds number ${ }^{1}$. For small values of the Gurzhi number the electrons propagate ballistically with a flat velocity profile across the bulk of the sample, while large values of $\mathrm{Gu}$ correspond to a Poiseuille-like flow with a nonuniform velocity distribution. This behavior is illustrated in Fig. 2, where we show velocity profiles for various values of Gu measured in a strip of width $W=5 \mu \mathrm{m}$.

The nonlocal effects in the electronic flow in graphene are typically studied in a Hall bar geometry $\frac{1115 \mid 20}{2}$. The inviscid, Ohmic current is expected to flow directly from the source to drain contact $\$ 38141$, while exponentially decaying into the bulk of the Hall bar. In long mesoscopic systems (i.e. with $L>W$ ) such decay is governed by the sample geometry rather than momentum relaxation as follows from the van der Pauw theorem 24/42/43. Our numerical solution to Eq. (1) demonstrates exactly this behavior, see Figs. 3 and 4 The top panel in Fig. 3 shows the flow pattern of the Ohmic flow across a Hall bar. Measuring the voltage drop (i.e., calculating the chemical potential difference) at a distance $x$ away from the source and drain contacts yields an exponentially decaying signal as illustrated in Fig. 4. We have verified that the decay length $\xi$ (defined in the figure caption) is mostly determined by the width of the Hall bar in agreement with the standard expectation based on the van der Pauw method ${ }^{24 / 43}$ and remains largely insensitive to 

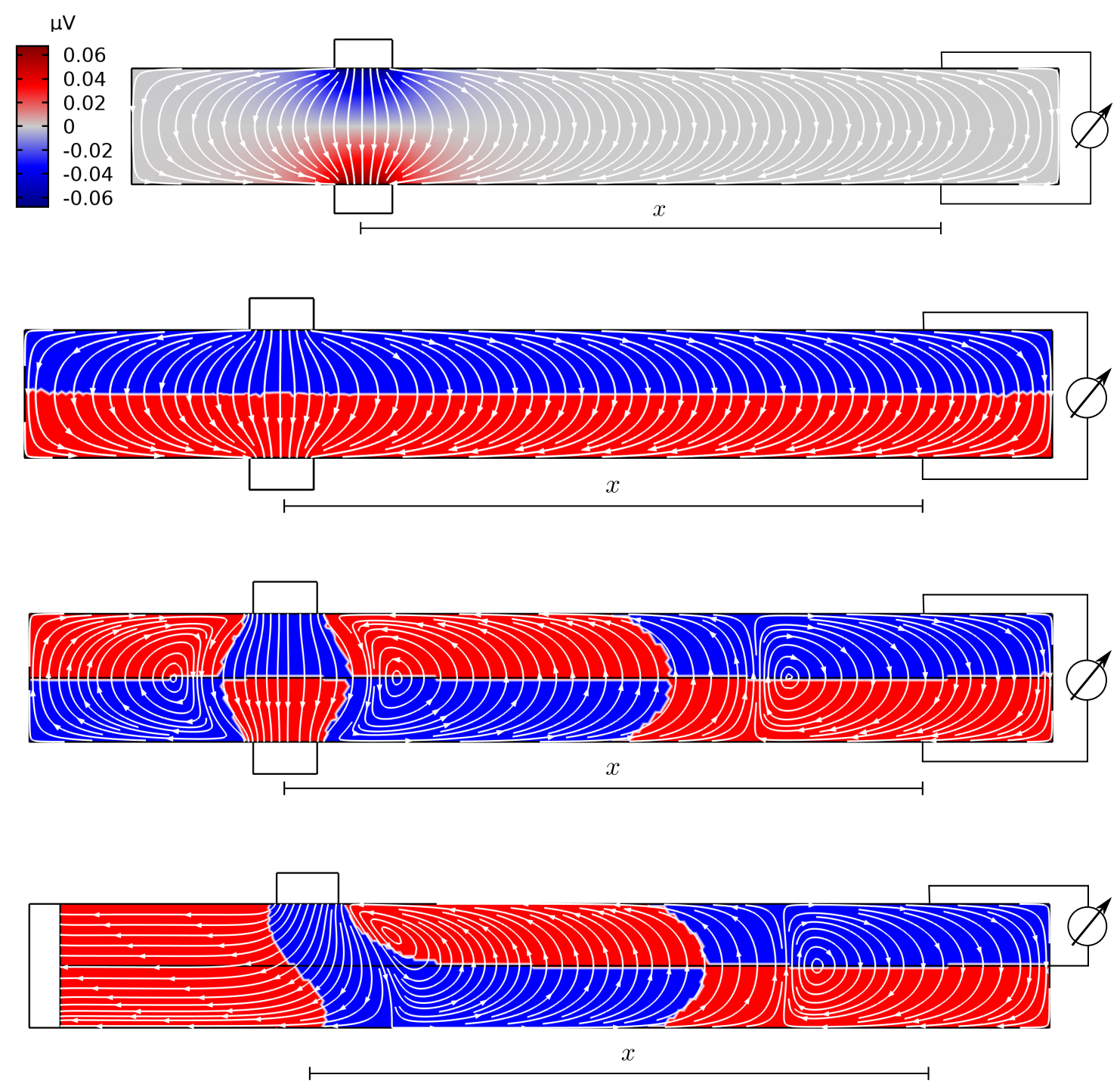

FIG. 3: Hydrodynamic electron flow in a Hall bar. Top two panels: Ohmic flow. Two bottom panels: viscous flow exhibiting multiple vortices and sign-alternating nonlocal resistance. White rectangles indicate the source and drain contacts. The data were calculated with the "slip boundary condition" Eq. B1 with the slip length ${ }^{11} \zeta=0.1 \mu \mathrm{m}$ and for $W=2 \mu \mathrm{m}, L=16 \mu \mathrm{m}$, $T=200 \mathrm{~K}, n=10^{12} \mathrm{~cm}^{-2}$, and $\mathrm{Gu}=0.175$ (increasing $\mathrm{Gu}$ or decreasing $\zeta$ reduces the vortex size increasing the number of vortices in the sample). The color map in the top panel shows the deviation of the electrochemical potential from its median value, while the red and blue colors in all other panels indicate the sign of this quantitiy (the broken white line separating the red and blue regions indicates the median value of the electrochemical potential; its patchiness reflects the finite precision of the simulation). The equidistant streamlines mask the exponential decay of the flow velocity, see Fig. 4.

a variation of either $L$ or $\ell_{\text {dis }}$.

The color map in the top panel in Fig. 3 shows the electrochemical potential distribution in the Hall bar. Away from the source and drain contacts, the electrochemical potential decays exponentially maintaining its sign along the Hall bar. To illustrate this point, the second panel in Fig. 3 shows the sign of the deviation of the electrochemical potential from its median value. The nonlocal resistance (at a distance $x$ away from the source and drain contacts) is exponentially small, see Fig. 4 , and positive.

Viscous flow in the Hall bar geometry is accompanied by formation of vortices ${ }^{20|29| 30 \mid 41}$ (also referred to as eddies or whirlpools). In traditional hydrodynamics 1 , vorticity appears already at the level of the Euler equation (describing ideal, inviscid flows), while viscosity tends to suppress it. Laminar flows (with small Reynolds number) tend to be vortex-free far away from flow boundaries or obstacles with the latter being "responsible" for nontrivial flow patterns (e.g., in the laminar wake ${ }^{1}$ ).

Now, electron motion in mesoscopic samples almost always takes place in proximity of the sample boundaries and hence the appearance of nontrivial vortex structure 


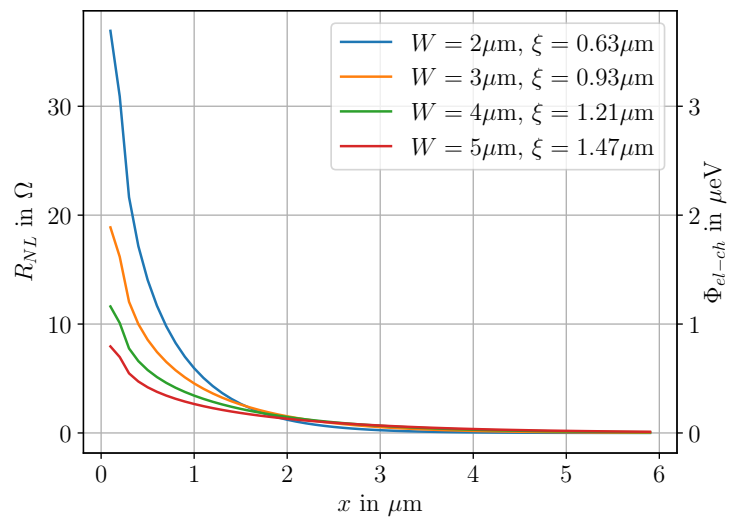

FIG. 4: Signal decay in the Ohmic flow (shown in the top panel in Fig. 3). The nonlocal resistance measured at a distance $x$ away from the source and drain contacts decays exponentially, $R_{N L} \propto \exp (-x / \xi)$. The obtained values of $\xi$ for different widths $W$ (shown in the legend) roughly agree with the estimate $\xi=W / \pi$ based on the van der Pauw method ${ }^{24 / 43}$.

is to be expected. Earlier measurements aiming at detection hydrodynamic flows of electrons in graphene ${ }^{20}$ used the nonlocal resistance $R_{N L}$ as the tool to uncover nontrivial flow patterns: a change of direction of the electronic flow may lead to a sign change in $R_{N L}$. The negative nonlocal resistance observed in these experiments was then interpreted ${ }^{29130 \mid 41}$ as a sign of vortex formation indicating a viscous flow. Later on it became clear that negative nonlocal resistance may appear in multiterminal measurements on ballistic (and perhaps even diffusive ${ }^{24}$ ) electronic systems ${ }^{11|15| 28}$ such that additional measurements were necessary to ensure that one observes indeed the hydrodynamic behavior.

We have solved the hydrodynamic equation (1) with the slip boundary conditions ${ }^{11140}$ in a somewhat longer (in comparison to the experimental samples of Refs. 14, 15|20), gated $\frac{15|20| 44 \mid 45}{}$ Hall bar. The results of our calculations are shown in the two bottom panels in Fig. 3 and in Fig. 5. The third panel in Fig. 3 shows the flow pattern in the "theoretical" geometry considered in Refs. 2941 with the source and drain contacts located on the opposite sides of the Hall bar. The bottom panel in Fig. 3 shows the current distribution in the "experimental" geometry of Ref. 20 (see also Ref. 30). In both cases, the flow pattern on the right of the source contact (where we calculate the nonlocal resistance) is rather similar. The first vortex appearing to the right of the source contact is located at a distance roughly equal to the width of the Hall bar ${ }^{29}$ and is somewhat asymmetric stretching more in the direction away from the contact. In a shorter Hall bar (as in the left side of the third panel in Fig. 3) there would be space for only one vortex, but in a longer system the second vortex appears. The size of the vortices and the exact location of the second vortex is determined by the interplay of the sample geom-

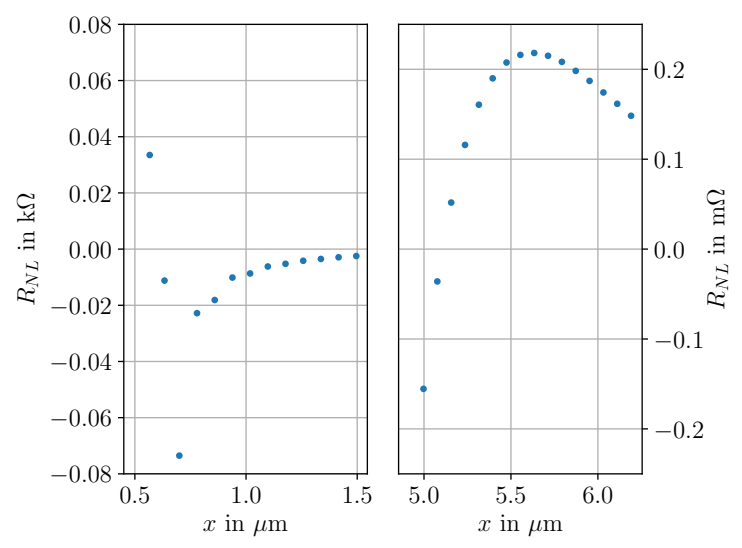

FIG. 5: Sign-alternating nonlocal resistance in viscous electronic flow (see the two bottom panels in Fig. 3). While still decaying exponentially, the nonlocal resistance measured in a viscous flow exhibiting multiple vortices changes sign as a function of the distance $x$ away from the source and drain contacts. Left panel: the first sign change in $R_{N L}$ due to the first vortex observed in Ref. 20 and calculated in Ref. 29. Right panel: the second sign change in $R_{N L}$ due to the second vortex with vorticity opposite to that of the first vortex. While the values of $R_{N L}$ are exponentially smaller, they remain in the measurable $\mathrm{m} \Omega$ range.

etry, slip length (see Appendix B), and disorder mean free path (we chose $\mathrm{Gu}=0.175$ on the basis of experimental data $11|13| 20$; increasing the value of $\mathrm{Gu}$ or reducing the slip length reduces the vortex size; this way we may observe more vortices in the sample). As the second vortex "spins" in the opposite direction to the first one, the resulting nonlocal resistance exhibit another sign change, see Fig. 5. Since the relation between the flow velocity and electrochemical potential involves derivatives, the sign change is not aligned with the vortex boundaries (such that the correspondence between the flow direction and the sign of $R_{N L}$ is not direct).

Given that the decay of the electronic flow (see Fig. 4) has mostly geometric origin 24|42|43, it is not surprising that the hydrodynamic flow decays in a similar fashion. As a result, the nonlocal resistance after the second sign change (see the right panel in Fig. 5) is several orders of magnitude smaller than the absolute value of the negative $R_{N L}$ after the first sign change. Nevertheless, using realistic parameters (with the values borrowed from the experiments of Refs. 11]13|15|20) we obtain values of order $m \Omega$ (measurable in a modern laboratory).

To conclude, we have solved the hydrodynamic equations in doped graphene in experimentally relevant Hall bar geometry. We have mapped the nontrivial vortex pattern of the viscous electronic flow and calculated the corresponding sign-alternating nonlocal resistance. In contrast to Ref. 33, this behavior is not related to quasiparticle recombination (which is only important in close proximity to the Dirac point) and magnetic field (see also Ref (46). At charge neutrality, the electric current 
density in the absence of magnetic field is decoupled from the hydrodynamic velocity, see Eq. A3. As a result, transport properties of neutral graphene are more complicated $12145 \sqrt[48]{4}$ and require further investigation. We believe that the obtained flow patterns are unlikely to occur in ballistic electronic systems. We expect that an experimental observation of multiple vortices with the help of modern imaging techniques of Refs. 7/11/12 combined with the measurement of the sign-alternating nonlocal resistance will uniquely identify the hydrodynamic behavior of charge carriers in graphene, as well as any other electronic system that is pure enough to support the "hydrodynamic temperature window" [based on the universality of the hydrodynamic equation (1) which unlike Eq. A1 - is no longer specific to Dirac fermions in graphene].

\section{Acknowledgments}

The authors wish to thank I.V. Gornyi, A.D. Mirlin, J. Schmalian, J.A. Sulpizio, M. Schütt, and A. Shnirman for fruitful discussions. This work was supported by the German Research Foundation DFG within FLAGERA Joint Transnational Call (Project GRANSPORT), by the European Commission under the EU Horizon 2020 MSCA-RISE-2019 program (Project 873028 HYDROTRONICS), and the MEPhI Academic Excellence Project, Contract No. 02.a03.21.0005.

\section{Appendix A}

The hydrodynamic description of electronic transport in graphene was discussed in detail in Refs. 31132 (see also Refs. (819). In the absence of the magnetic field the generalized Navier-Stokes equation in graphene is

$$
\begin{aligned}
\mathcal{W}\left(\partial_{t}+\boldsymbol{u} \cdot \boldsymbol{\nabla}\right) \boldsymbol{u}+v_{g}^{2} \boldsymbol{\nabla} P & +\boldsymbol{u} \partial_{t} P+e(\boldsymbol{E} \cdot \boldsymbol{j}) \boldsymbol{u}= \\
& =v_{g}^{2}[\eta \Delta \boldsymbol{u}+e n \boldsymbol{E}]-\boldsymbol{j}_{E} / \tau_{\mathrm{dis}}
\end{aligned}
$$

where $\eta$ is the shear viscosity $1420134, n$ is the carrier density, $\boldsymbol{E}$ is the electric field, $v_{g}$ is the Fermi velocity in graphene, and $\tau_{\text {dis }}$ is the disorder mean free time. The enthalpy $\mathcal{W}$ and pressure $P$ are related to the energy density $n_{E}$ in graphene by the "equation of state" 3132

$$
\mathcal{W}=n_{E}+P=3 n_{E}\left(2+u^{2} / v_{g}^{2}\right)^{-1} .
$$

The carrier and energy currents $\left(\boldsymbol{j}\right.$ and $\boldsymbol{j}_{E}$, respectively) are related to the hydrodynamic velocity $\boldsymbol{u}$ as

$$
\boldsymbol{j}=n \boldsymbol{u}+\delta \boldsymbol{j}, \quad \boldsymbol{j}_{E}=\mathcal{W} \boldsymbol{u},
$$

where $\delta \boldsymbol{j}$ is the dissipative correction 949 . The full electric current is given by $\boldsymbol{J}=e \boldsymbol{j}$. The hydrodynamic theory is completed by the continuity equations

$$
\partial_{t} n+\nabla_{\boldsymbol{r}} \cdot \boldsymbol{j}=0
$$

$$
\partial_{t} n_{E}+\nabla_{\boldsymbol{r}} \cdot \boldsymbol{j}_{E}=e \boldsymbol{E} \cdot \boldsymbol{j}
$$

where the last term describes Joule heat. The continuity equation $\mathrm{A} 4 \mathrm{a}$ is valid in any electronic system, while Eq. A4b neglects possible energy losses due to coupling to collective excitations (e.g., phonons, plasmons, etc.).

Consider a graphene sample away from charge neutrality, i.e. the system considered in the imaging experiments of Refs. 711, In this case the densities are determined by the chemical potential $\mu$

$$
n(\mu \gg T)=\frac{\mu^{2}}{\pi v_{g}^{2}}, \quad n_{E}(\mu \gg T)=\frac{2 \mu^{3}}{3 \pi v_{g}^{2}}, \quad \delta \boldsymbol{j}=0 .
$$

Similarly, the mean free time assumes the value $\tau_{\text {dis }}(\mu)$. Within linear response and in the case of stationary flow, the Navier-Stokes equation (A1) takes the form (1)

$$
v_{g}^{2} \boldsymbol{\nabla} P=v_{g}^{2}[\eta \Delta \boldsymbol{u}+e n \boldsymbol{E}]-\mu n \boldsymbol{u} / \tau_{\text {dis }}
$$

In the rest of the paper we analyze this equation numerically and establish the local flow patterns in experimentally relevant geometries.

In the textbook case of a uniform current ${ }^{[5 / 9 / 32}$ we recover the standard Drude formula

$$
\boldsymbol{J}=e n \boldsymbol{u}=\left(e^{2} / \pi\right) \mu \tau_{\text {dis }} \boldsymbol{E} \Rightarrow \sigma=\left(e^{2} / \pi\right) \mu \tau_{\text {dis }} .
$$

Relating the pressure to the carrier density

$$
\nabla P=\frac{n}{\rho(0)} \nabla n, \quad \rho(0)=\frac{\partial n}{\partial \mu},
$$

where $\rho(0)$ is the density of states (DoS), and taking into account the local charge density variations in Eq. (1), one arrives at the usual description of the diffusive transport

$$
\boldsymbol{J}=\left(e^{2} / \pi\right) \mu \tau_{\text {dis }} \boldsymbol{E}-e D \nabla n, \quad D=v_{g}^{2} \tau_{\text {dis }} / 2,
$$

where $D$ is the diffusion coefficient (see Ref. 4, for a simplified discussion in a similar context see Ref. (50).

\section{Appendix B}

Our numerical technique of choice for an analytically intractable problem [e.g., the Navier-Stokes equation [A1] in a realistic geometry] is the finite element method. With this method we can calculate the velocity $\boldsymbol{u}$ and carrier density $n$ up to a finite accuracy, which is given by the number of elements in which we split the system. A solution can be approximated by an auxiliary function

$$
u_{h}(x)=\sum_{i=0}^{N} u_{i} \varphi_{i}(x)
$$

interpolating between the predetermined points separating the elements. The test function $\varphi_{i}(x)$ can be chosen arbitrarily provided it satisfies

$$
\varphi_{i}(x)=\left\{\begin{array}{ll}
1, & \text { if } x=x_{i} \\
0, & \text { if } x \neq x_{i}
\end{array} .\right.
$$


For our purposes, it was sufficient to choose a linear Lagrange polynomial for the carrier density and a quadratic one for the hydrodynamic velocity.

This approach can be applied to any linear differential equation of form

$$
\mathcal{L} u(x)=g(x),
$$

which can be solved up to a predetermined accuracy by building its weak form

$$
\sum_{i=0}^{N} u_{i} \int_{\Omega} \mathcal{L} \varphi_{i}(x) \varphi_{j}(x) d \Omega=\int_{\Omega} g(x) \varphi_{j}(x) d \Omega .
$$

This yields a linear system of equations with an invertable $(N+1) \times(N+1)$ matrix that allows one to find the $N+1$ vector $u_{i}$ at every point.

The Navier-Stokes equation A1 in graphene should be supplemented by the appropriate boundary conditions. In the case of the source and drain contacts we apply the finite Dirichlet and zero flux boundary conditions. For the edges of the graphene Hall bar we apply the slip boundary condition $\underline{40}$

$$
\left.u_{\alpha}^{t}\right|_{\partial \Omega}-\zeta \boldsymbol{n} \cdot \nabla u_{\alpha}^{t}=0
$$

where $\boldsymbol{n}$ is the unit vector normal to the boundary.

The boundary conditions have a profound effect on the resulting flow patterns 41 . For the commonly used "noslip" boundary conditions $(\zeta=0)$, the vortices shown in Fig. 3 exhibit the smallest tail (in the direction away from the source contact), which is growing with $\zeta$ (assuming all other parameters remain unchanged). In the opposite limit of "no-stress" boundary conditions $(\zeta \rightarrow \infty)$ the tail length exceeds all system sizes used in our simulations such that the flow pattern exhibits a single vortex ${ }^{29 / 41}$.

The rectangular contacts shown in Fig. 3 possess sharp corners. Taken literally, such geometry is plagued with singular behavior $29151 \sqrt[53]{ }$. Given that these singularities have nothing to do with the observed vorticity 41 (in the context of the Hall effect the corners in real devices are known to be effectively rounded ${ }^{52 / 53}$ ) we smooth out the Dirichlet boundary condition at the corners of the source contact to obtain stable flow patterns.
1 L. D. Landau and E. M. Lifshitz, Fluid Mechanics (Pergamon Press, London, 1959).

2 P. M. Chaikin and T. C. Lubensky, Principles of Condensed Matter Physics (Cambridge University Press, 1995).

3 D. Vollhardt and P. Wölfle, The Superfluid Phases of Helium 3 (Taylor and Francis, London, 1990).

${ }^{4}$ E. M. Lifshitz and L. P. Pitaevskii, Physical Kinetics (Pergamon Press, London, 1981).

5 J. M. Ziman, Principles of the Theory of Solids (Cambridge University Press, Cambridge, 1965).

${ }^{6}$ Y. Imry, Introduction to Mesoscopic Physics (Oxford University Press, Oxford, 2008).

7 L. Ella, A. Rozen, J. Birkbeck, M. Ben-Shalom, D. Perello, J. Zultak, T. Taniguchi, K. Watanabe, A. K. Geim, S. Ilani, et al., Nat. Nanotechnol. 14, 480 (2019).

8 B. N. Narozhny, I. V. Gornyi, A. D. Mirlin, and J. Schmalian, Annalen der Physik 529, 1700043 (2017).

9 A. Lucas and K. C. Fong, J. Phys: Condens. Matter 30, 053001 (2018).

10 R. N. Gurzhi, Soviet Physics Uspekhi 11, 255 (1968).

11 J. A. Sulpizio, L. Ella, A. Rozen, J. Birkbeck, D. J. Perello, D. Dutta, M. Ben-Shalom, T. Taniguchi, K. Watanabe, T. Holder, et al., Nature 576, 75 (2019).

12 M. J. H. Ku, T. X. Zhou, Q. Li, Y. J. Shin, J. K. Shi, C. Burch, H. Zhang, F. Casola, T. Taniguchi, K. Watanabe, et al. (2019), arXiv:1905.10791.

13 P. Gallagher, C.-S. Yang, T. Lyu, F. Tian, R. Kou, H. Zhang, K. Watanabe, T. Taniguchi, and F. Wang, Science 364, 158 (2019).

14 A. I. Berdyugin, S. G. Xu, F. M. D. Pellegrino, R. Krishna Kumar, A. Principi, I. Torre, M. B. Shalom, T. Taniguchi, K. Watanabe, I. V. Grigorieva, et al., Science 364, 162 (2019).

15 D. A. Bandurin, A. V. Shytov, L. S. Levitov, R. Krishna
Kumar, A. I. Berdyugin, M. Ben Shalom, I. V. Grigorieva, A. K. Geim, and G. Falkovich, Nat. Commun. 9, 4533 (2018).

16 B. A. Braem, F. M. D. Pellegrino, A. Principi, M. Röösli, C. Gold, S. Hennel, J. V. Koski, M. Berl, W. Dietsche, W. Wegscheider, et al., Phys. Rev. B 98, 241304(R) (2018).

17 A. Jaoui, B. Fauqué, C. W. Rischau, A. Subedi, C. Fu, J. Gooth, N. Kumar, V. Süß, D. L. Maslov, C. Felser, et al., npj Quantum Materials 3, 64 (2018).

18 P. J. W. Moll, P. Kushwaha, N. Nandi, B. Schmidt, and A. P. Mackenzie, Science 351, 1061 (2016).

19 J. Crossno, J. K. Shi, K. Wang, X. Liu, A. Harzheim, A. Lucas, S. Sachdev, P. Kim, T. Taniguchi, K. Watanabe, et al., Science 351, 1058 (2016).

20 D. A. Bandurin, I. Torre, R. Krishna Kumar, M. Ben Shalom, A. Tomadin, A. Principi, G. H. Auton, E. Khestanova, K. S. Novoselov, I. V. Grigorieva, et al., Science 351, 1055 (2016).

${ }^{21}$ R. V. Gorbachev, J. C. W. Song, G. L. Yu, A. V. Kretinin, F. Withers, Y. Cao, A. Mishchenko, I. V. Grigorieva, K. S. Novoselov, L. S. Levitov, et al., Science 346, 448 (2014).

22 W. J. Skocpol, P. M. Mankiewich, R. E. Howard, L. D. Jackel, D. M. Tennant, and A. D. Stone, Phys. Rev. Lett. 58, 2347 (1987).

23 A. K. Geim, P. C. Main, P. H. Beton, P. Steda, L. Eaves, C. D. W. Wilkinson, and S. P. Beaumont, Phys. Rev. Lett. 67, 3014 (1991).

24 G. Mihajlović, J. E. Pearson, M. A. Garcia, S. D. Bader, and A. Hoffmann, Phys. Rev. Lett. 103, 166601 (2009).

${ }^{25}$ H. van Houten, C. W. J. Beenakker, J. G. Williamson, M. E. I. Broekaart, P. H. M. van Loosdrecht, B. J. van Wees, J. E. Mooij, C. T. Foxon, and J. J. Harris, Phys. Rev. B 39, 8556 (1989).

${ }^{26}$ K. L. Shepard, M. L. Roukes, and B. P. van der Gaag, Phys. Rev. Lett. 68, 2660 (1992). 
27 Y. Hirayama, A. D. Wieck, T. Bever, K. von Klitzing, and K. Ploog, Phys. Rev. B 46, 4035 (1992).

28 A. V. Shytov, J. F. Kong, G. Falkovich, and L. S. Levitov, Phys. Rev. Lett. 121, 176805 (2018).

29 L. S. Levitov and G. Falkovich, Nat. Phys. 12, 672 (2016).

30 I. Torre, A. Tomadin, A. K. Geim, and M. Polini, Phys. Rev. B 92, 165433 (2015).

31 U. Briskot, M. Schütt, I. V. Gornyi, M. Titov, B. N. Narozhny, and A. D. Mirlin, Phys. Rev. B 92, 115426 (2015).

32 B. N. Narozhny, Annals of Physics 411, 167979 (2019).

${ }^{33}$ H.-Y. Xie and A. Levchenko, Phys. Rev. B 99, 045434 (2019).

34 B. N. Narozhny and M. Schütt, Phys. Rev. B 100, 035125 (2019).

35 M. Shavit, A. V. Shytov, and G. Falkovich, Phys. Rev. Lett. 123, 026801 (2019).

${ }^{36}$ M. S. Foster and I. L. Aleiner, Phys. Rev. B 79, 085415 (2009).

37 R. Moessner, N. Morales-Durán, P. Surówka, and P. Witkowski, Phys. Rev. B 100, 155115 (2019).

${ }^{38}$ H. Guo, E. Ilseven, G. Falkovich, and L. S. Levitov, Proceedings of the National Academy of Sciences 114, 3068 (2017).

39 P. S. Alekseev, A. P. Dmitriev, I. V. Gornyi, V. Y. Kachorovskii, B. N. Narozhny, and M. Titov, Phys. Rev. B 97, 085109 (2018).

${ }^{40}$ E. I. Kiselev and J. Schmalian, Phys. Rev. B 99, 035430
(2019).

41 G. Falkovich and L. S. Levitov, Phys. Rev. Lett. 119 , 066601 (2017).

42 L. J. van der Pauw, Philips Tech. Rev. 20, 223 (1958).

43 D. A. Abanin, S. V. Morozov, L. A. Ponomarenko, R. V. Gorbachev, A. S. Mayorov, M. I. Katsnelson, K. Watanabe, T. Taniguchi, K. S. Novoselov, L. S. Levitov, et al., Science 332, 328 (2011).

44 I. L. Aleiner and B. I. Shklovskii, Phys. Rev. B 49, 13721 (1994)

45 P. S. Alekseev, A. P. Dmitriev, I. V. Gornyi, V. Y. Kachorovskii, B. N. Narozhny, M. Schütt, and M. Titov, Phys. Rev. Lett. 114, 156601 (2015).

46 S. Danz, M. Titov, and B. N. Narozhny (2019), arXiv:1912.12341.

47 B. N. Narozhny, I. V. Gornyi, M. Titov, M. Schütt, and A. D. Mirlin, Phys. Rev. B 91, 035414 (2015).

48 P. S. Alekseev, A. P. Dmitriev, I. V. Gornyi, V. Y. Kachorovskii, B. N. Narozhny, M. Schütt, and M. Titov, Phys. Rev. B 95, 165410 (2017).

49 B. N. Narozhny, Phys. Rev. B 100, 151434 (2019).

50 B. N. Narozhny, I. L. Aleiner, and A. Stern, Phys. Rev. Lett. 86, 3610 (2001).

51 R. F. Wick, Journal of Applied Physics 25, 741 (1954).

${ }^{52}$ R. W. Rendell and S. M. Girvin, Phys. Rev. B 23, 6610 (1981).

53 S. Kawaji, Surface Science 73, 46 (1978). 